Documento de Trabajo 2005-03

\author{
Facultad de Ciencias Económicas y Empresariales
}

Universidad de Zaragoza

\title{
Detecting Determinism Using Recurrence Quantification Analysis: Three Test Procedures
}

\author{
$\mathbf{M}^{\mathrm{a}}$ Teresa Aparicio \\ Eduardo F. Pozo \\ Dulce Saura \\ Departamento de Análisis Económico \\ Facultad de Ciencias Económicas y Empresariales \\ (Universidad de Zaragoza) \\ Dirección: Facultad de CC. EE y EE., Gran Vía 2, 50005 Zaragoza, España. \\ Correo electrónico: aparicio@unizar.es

\begin{abstract}
In this paper we use the "Recurrence Quantification Analysis” proposed by Zbilut and Webber (1992), to develop three test procedures that allow us to detect determinism underlying series. Based on the concept of "Percent of Determinism" we obtain three statistics that allow us to test for the null hypothesis of randomness. The results of the application of these procedures to a set of simulated and real series reveal good behaviour in a wide variety of situations, including different sample sizes and series affected by noise.
\end{abstract}

Keywords: Recurrence Quantification Analysis, Test for determinism, Nonlinear dynamics.

Acknowledgements: This research was supported by the BEC2003-02827 project. 


\section{1.- Introduction}

The study of time series with the aim of trying to characterise the underlying dynamic system (or data generating process) is a field of study of great interest in economics that can be undertaken from two different perspectives.

The first focuses on the area of lineal dynamics, justifying the irregular oscillations from the existence of exogenous random shocks that do not necessarily have an economic explanation. The poor predictive results obtained through this approach gave place to a second focus that considers that the fluctuations of the series respond to a non-linear structure. This non-linearity can affect the random part of the process (specifications ARCH, GARCH......) or the systematic part, in such a way that a series that is apparently random could have been generated by a low dimension deterministic system. The possibility that oscillations of economic series may be caused by non-linear deterministic relationships rather than random shocks opens new directions in the explanation and forecasting of economic phenomena.

The starting point for this type of study comes from the so-called chaotic dynamics or deterministic chaos. In practice, different methods have been proposed with the aim of testing whether a series may or may not have been generated within the framework of a chaotic system. Among the more widely used we can highlight: the Grassbeger-Procaccia algorithm, the Lyapunov exponents, the BDS test, etc. Most of these procedures are based on the so-called "Time Delay Method", through which the original series is transformed into an n-dimensional system which, depending on the fulfilment of certain conditions, is topologically equivalent to the original system from which the series was supposedly determined; (see Packard et al., 1980 and Takens, 1981). As well as the characteristic conditions of the "Time Delay Method", some of these procedures require the prior determination of certain parameters and the fulfilment of restrictive requirements such as a high sample size, the absence of noise, the stationarity of the series, etc.

In a paper published in 1987, Eckmann et al. presented a graphic procedure, which they called "recurrence plot" (RP), to detect patterns of recurrence in the data, which are one of the most important characteristics of dynamic systems. In the context 
of chaotic dynamics, this procedure, even though it is also based on the "Time Delay Method", has some advantages over the previous ones. Among them, we can highlight its apparent simplicity of implementation and interpretation and the fewer prior requirements on the data base that it needs. ${ }^{1}$ However, being a tool of a visual and qualitative nature, its results are not always conclusive and so it ended up being considered a minor technique or, at best, one to be applied prior to others that allowed more robust conclusions.

Zbilut and Webber (1992) and Webber and Zbilut (1994) recognised this drawback and tried to overcome it through what they called Recurrence Quantification Analysis (RQA). The RQA considers that it is possible to quantify the information supplied by the RP and, using certain simple pattern recognition algorithms, to summarise the information in a series of indicators or statistics. We believe that this technique is of great interest in that it allows us to make use of the advantages of the RP and obtain more objective information than that which could be derived from a purely visual analysis.

The objective of this paper is twofold. In the first place, to obtain a clearer interpretation of the results of one of the RQA indicators, precisely the so-called percent of determinism (\%DET). As we later justify, we understand that this indicator is the most important when determining the random or deterministic character of the series being studied. Secondly, to apply the RQA to economic series with the aim of providing additional evidence about the possibilities of using the method in this field.

The structure of the present paper is as follows. In Section 2 the RP and the basic ideas related to the RQA are introduced. Section 3 focuses on the study of the \%DET statistic, on the basis of which a testing strategy is defined that allows us to establish the random or deterministic character of the series. In Section 4, the procedure is applied to a number of real and simulated series with the aim of evaluating its adequacy. Finally, Section 5 presents the main conclusions obtained.

\footnotetext{
${ }^{1}$ Some authors emphasize the advantages of this technique even more. For example, Casdagli (1997) affirms that techniques based on recurrence plots provide a unified framework for addressing the principal objectives of nonstationary time series analysis: characterization, prediction, change point detection and hypothesis testing.
} 


\section{2.- Recurrence Plots And Recurrence Quantification Analysis}

As is normally the case in most chaotic dynamics techniques, the starting point of the RPs is based on the Time Delay Method through which the original series is transformed into a set of $m$-tuples whose co-ordinates are the present and lagged values of the series. This $m$-dimensional embedding requires the specification of the values of two parameters: the embedding dimension $(m)$ and the time delay or time lag. We will return to the determination of these parameters at the end of the section.

An RP is a two-dimensional representation of the m-dimensional embedding of the data that is obtained by plotting a point on the $\mathrm{i}-\mathrm{j}$ plane if vector $\mathrm{x}(\mathrm{i})$ is close to vector $x(j)$. It is necessary to define a threshold or distance " $r$ " which determines the criterion of closeness. With regard to this, the literature proposes various strategies which can be classified into two groups:

1.-To consider " $\mathrm{r}$ " constant, either as a proportion of the standard deviation (Casdagli, 1997) or as a proportion of the average distance of the data (Zbilut and Webber, 1992).

2.-To consider " $\mathrm{r}$ " variable, with the requirement that a minimum number of points enter the neighbourhood of each vector x(i); (Eckmann et al., 1987).

This graphic tool shows different structures depending on the nature of the series under study. In particular, it is capable of detecting the time recurrence patterns underlying deterministic systems (whether they are chaotic or not). Non-chaotic deterministic systems exhibit very simple regular structures, while the RPs of chaotic systems also show a certain regularity but with more complex and denser features. On the other hand, the RPs obtained from purely random systems do not show distinguishable patterns, appearing as a cloud of points with no apparent structure.

\section{(Figure 1 about here)}

RPs have been used as a diagnostic tool of time series in various fields, principally in natural sciences (Physics, Chemistry, Biology......). However, being a graphic tool, its interpretation is obviously, on many occasions, complicated. This problem is further accentuated when we wish to apply it in social sciences such as 
Economics in which the available data series are not usually very long and, presumably, in most cases are affected by some type of noise. To overcome these inconveniences, RQA ("Recurrence Quantification Analysis") has the objective of quantifying the information supplied by the RPs and elaborating a series of quantitative indicators that allow us to interpret this information more rigorously. The starting point of the RQA is the following function:

$$
\mathrm{d}_{\mathrm{ij}}= \begin{cases}1, & \text { if }\|x(\mathrm{i})-\mathrm{x}(\mathrm{j})\|<\mathrm{r} \\ 0, & \text { if }\|\mathrm{x}(\mathrm{i})-\mathrm{x}(\mathrm{j})\| \geq \mathrm{r}\end{cases}
$$

Clearly, the points of the RP correspond to the values of $d_{i j}=1$. Therefore, this indicator function collects the information given by the RP in a binary symmetric $n \times n$ matrix (which we will call "recurrence matrix", RM), with $n$ being the number of vectors of the embedding. The main features of the structure of the RP may be quantified from this matrix and transformed into analytical indicators. Among the various patterns which can be recognised from the RM, the RQA focuses its attention on the points forming lines parallel to the main $45^{\circ}$ diagonal. These lines may correspond to two situations:

a)Recurrent points of two parts of the trajectory which keep close together for a while, such as the points in Figure 2-(a). ${ }^{2}$ This pattern is related to the concept of spatial correlation, characteristic of the behaviour of chaotic systems.

b)Consecutive recurrent points, caused by the existence of time correlation within the trajectory. These points form segments on the diagonal lines closest to the main diagonal. For example, the points in Figure 2-(b).

The presence of such diagonal structuring in the RM is assumed to be a distinctive feature of deterministic systems.

There are other kinds of lines appearing in the RM, which may also give useful information:

-Patterns along the $135^{\circ}$ diagonal, which could correspond to the existence of false neighbours in the embedding, i.e. consecutive points of "false recurrence" because of a small embedding dimension. In other words, points which are close in the

\footnotetext{
${ }^{2}$ The elements of the embedding are obtained from a time series. For this reason we consider that they determine a "trajectory".
} 
projection onto a space with an insufficient dimension, but that would not be for the true dimension of the system. See Figure 2-(c).

-Horizontal and vertical lines, formed by consecutive points of the embedding which are all close to a certain point, such as the points in Figure 2-(d). These lines may be helpful to detect structural changes in the dynamics of the system (such as periodicchaos and chaos-chaos transitions). See Gao and Cai (2000) and Marwan et al. (2002).

(Figure 2 about here)

Finally, considering that the RM is symmetric, the analytical indicators introduced by the RQA are obtained in practice using the upper (or lower) triangular part of the RM excluding the main diagonal (from now on, TRM). ${ }^{3}$

The main statistics proposed by the RQA are the following:

a) \%REC: Percent of recurrence or global recurrence measure, defined as:

$$
\% R E C=100 \times \frac{N R E C}{N P} \text {, where: }
$$

NREC is the number of recurrent points or number of ones of the TRM and NP is the total elements of the TRM. Obviously,

$$
N P=\frac{n \cdot(n-1)}{2}
$$

b) \%DET: Percent of determinism.

$$
\% D E T=100 \times \frac{N P D}{N R E C} \text {, where: }
$$

$N P D$ is the number of points on lines parallel to the main diagonal, considering that a line is formed with a minimum of at least two adjacent points.

c) ENT: Shannon entropy or a measure of the average information contained in the line-segment distribution.

$$
E N T=-\sum_{k=1}^{n-1} p_{k} \cdot \log _{2}\left(p_{k}\right) \text {, where: }
$$

\footnotetext{
${ }^{3}$ All the elements on the main diagonal will be ones (each vector will always be "close" to itself). In other words, points there are trivially recurrent and, consequently, excluded from the analysis.
} 
$p_{k}=$ proportion of diagonal lines of length $k$ over the total diagonal lines, that is to say, the relative frequency of the lengths of diagonal lines. This statistic gives information about the diversity of lines parallel to the main diagonal. Hence it can also be an indicator of the deterministic or stochastic nature of the series.

d) DIV (Divergence): Reciprocal of the longest diagonal line, which sometimes is associated with the sensitive dependence on initial conditions. Hence, in chaotic systems it is assumed to be directly related to the maximum Lyapunov exponent (see Eckmann et all, 1987).

e) TREND: This statistic measures the stationarity of the data. It is calculated as the slope of the $\% R E C$ as a function of the displacement from the main $45^{\circ}$ diagonal.

As we said at the beginning of this section, the construction of the RPs and the obtaining of the RMs are carried out on the basis of the Time Delay Method which requires previously fixing the values of the embedding dimension and the time delay. In general, the results obtained from the application of the Time Delay Method are sensitive to the values chosen for these parameters, as can be gathered from the works of Haucke et al. (1986), Buzug et al. (1990) and Mayfield and Mizrach (1992). Within the framework of the RPs, even though Iwansky and Bradley (1998) and Bradley (2002) stated that these graphics were independent from these parameters, that is to say, they would remain qualitatively stable when the parameters change, other investigations have shown strong evidence to the contrary. This is the case of Atay and Altintas (1999), who even show how it is possible to use the information given by the RPs and the RMs to try to establish adequate values of the embedding dimension and the time delay. Gao and Cai (2000) come to the same conclusion, based on the interpretation of the different types of lines that can appear in an RP. Thiel et al. (2004) conclude that "both groups are in some sense right", showing that some indicators obtained from the RPs are independent from the embeddings while others are not.

\section{3.- Applications of The RQA: Stochastic Versus Deterministic Series.}

As we have said above, within the framework of RQA, the essential feature that characterises deterministic processes is the existence of lines parallel to the main diagonal. The \%DET, DIV and ENT statistics described in Section 2 are based precisely 
on this fact. So, the greater the number of points on line segments, the greater the deterministic component of the series will be. ${ }^{4}$ As well as the three statistics mentioned above (especially the \%DET, which is the one that is most clearly focused on this characteristic), Atai and Altintas (1999) use the “average line length” $(A L L)^{5}$, whose calculation and interpretation is similar to the \%DET although, in our opinion, it has an important advantage: this indicator considers the length of the different lines whereas the $\% D E T$ counts all the points on the parallel lines equally, regardless of their size.

In general terms, for purely random processes the value of the \%DET would be very small (close to zero) ${ }^{6}$ while processes with some deterministic component would be associated with values of this statistic significantly greater than zero. Equally, the bigger the ALL, the greater the evidence that the underlying process is deterministic. ${ }^{7}$

However, these interpretations, very clear for extreme values, are not so obvious if the result has an intermediate value. For example, the values of \%DET and $A L L$ associated with the RPs of Figure 1 are shown in Table 1:

\section{(Table 1 about here)}

We understand, therefore, that it would be very useful to have some test that would allow us to decide which values of these indicators should be considered small and which should be considered high. In other words, what is, in each case, the cut-off point that determines whether the series comes from a random or deterministic system. To try to give a solution to this problem is the main objective of this paper.

As a starting point, we are going to define a random variable, $\mathrm{L}$, that assigns to each element of the TRM, $y_{j}$, the size of the line ${ }^{8}$ that this element belongs to, $L\left(y_{j}\right)$. Given that $L=1, \ldots ., n-1$, this random variable can take $n-1$ different values.

From the geometrical structure of the TRM, we are going to obtain the probability distribution of $L$ for the case in which the data generating process is

\footnotetext{
${ }^{4}$ Zbilut et al. (2000) specifically use this characteristic as a simple empirical deterministic test.

${ }^{5}$ Average length (i.e. average number of points) of the lines parallel to the main diagonal.

${ }^{6}$ One could think that, in this case, the resulting value of the \%DET should be zero. However, this is not necessarily so: a purely random series would give rise to an RP with randomly distributed points, some of which could form diagonal lines, i.e. producing positive values of the \%DET.

${ }^{7}$ To calculate the $A L L$ we will consider that the points that do not belong to lines parallel to the main diagonal form lines of length 1 . So this indicator will be always greater or equal to one.

${ }^{8}$ From now on, when we talk of lines of the TRM, we always mean lines parallel to the main diagonal.
} 
absolutely random (which would correspond to a totally random disposition of points within the plot, or, in other words, to a TRM formed by arbitrarily distributed ones and zeros). In these conditions, the probability that a point belongs to a line of size " $L$ " will depend on the place this point occupies in the TRM. Below, we are going to specify the expressions of these probabilities. ${ }^{9}$

The simplest case consists of the recurrent isolated points, that is, recurrent points that do not form part of lines of a length equal or superior to 2 (in this context, we consider that these points form lines of a length equal to one). We can distinguish three types of lines of length 1 (see Figure 3):

That which the element $(1,1)$ of the TRM would form if it were a recurrent point. The probability of this happening will be:

Those corresponding to the other points in the first row and the first column of the TRM, 2.(n-2) in total. These points would form a line of length 1 if they were recurrent points and the contiguous point was not. ${ }^{10}$ Therefore the probability of one of these points forming a line of length 1 will be:

$$
\frac{N R E C}{N P} \cdot \frac{N P-N R E C}{N P-1} \text {. }
$$

Those that the other points of the TRM could form, (n-2) $(n-3) / 2$ in total. These points would form a line of length 1 if they were recurrent points and neither of the two adjacent points were. The probability of this happening will be:

$$
\frac{N R E C}{N P} \cdot \frac{N P-N R E C}{N P-1} \cdot \frac{N P-N R E C-1}{N P-2}
$$

(Figure 3 about here)

In consequence, the probability of a point of the TRM forming a line of a length equal to one will be:

\footnotetext{
${ }^{9}$ The study of these lines in terms of probability distribution is also used by Thiel et al. (2003) with the aim of estimating some dynamical invariants from RPs. Particularly, they focus on the probability to find a diagonal of at least length $l$ in the RP.

${ }^{10}$ Understanding by contiguous points those which are so in the direction of the main diagonal.
} 


$$
\begin{gathered}
\mathrm{P}(1)=\left[\frac{N R E C}{N P}+\left(\frac{N R E C}{N P} \cdot \frac{N P-N R E C}{N P-1}\right) \cdot 2 \cdot(n-2)+\right. \\
\left.+\left(\frac{N R E C}{N P} \cdot \frac{N P-N R E C}{N P-1} \cdot \frac{N P-N R E C-1}{N P-2}\right) \cdot \frac{(n-2) \cdot(n-3)}{2}\right] \cdot \frac{1}{N R E C}
\end{gathered}
$$

Reasoning in similar terms, we could obtain the probability of any point of the TRM belonging to a line of size 2. In this case, from the geometrical structure of the TRM we could distinguish three possible types of lines of length 2 (see Figure 4):

The line formed by the points $(1,2)$ and $(2,1)$ of the TRM. For these two points to form a line both must be recurrent. The probability of this happening will be:

$$
\frac{N R E C}{N P} \cdot \frac{N R E C-1}{N P-1}
$$

Those that contain elements of the first row or the first column of the TRM. The probability that two points of the TRM form a line of this type would be:

$$
\frac{N R E C}{N P} \cdot \frac{N R E C-1}{N P-1}
$$

there being $2 \cdot(n-3)$ of these lines possible.

The other lines of length 2, that is, lines formed by points not belonging to the first row or column of the TRM (“interior" points in terms of Figure 4(c)). We are talking of $\frac{(n-3) \cdot(n-4)}{2}$ possible lines, with the probability of each of them existing being:

$$
\frac{N R E C}{N P} \cdot \frac{N R E C-1}{N P-1} \cdot \frac{N P-N R E C}{N P-2} \cdot \frac{N P-N R E C-1}{N P-3}
$$

(Figure 4 about here)

Hence, the probability of a recurrent point being on a line with a length equal to 2 will be:

$$
\begin{aligned}
& \mathrm{P}(2)=2 \cdot\left[\frac{N R E C}{N P} \cdot \frac{N R E C-1}{N P-1}+\left(\frac{N R E C}{N P} \cdot \frac{N R E C-1}{N P-1} \cdot \frac{N P-N R E C}{N P-2}\right) \cdot 2 \cdot(n-3)+\right. \\
& \left.+\left(\frac{N R E C}{N P} \cdot \frac{N R E C-1}{N P-1} \cdot \frac{N P-N R E C}{N P-2} \cdot \frac{N P-N R E C-1}{N P-3}\right) \cdot \frac{(n-3) \cdot(n-4)}{2}\right] \cdot \frac{1}{N R E C}
\end{aligned}
$$

Reasoning in a similar way for lines of size 3, 4, etc., we would obtain the 
following general expression:

$$
\begin{aligned}
& \mathrm{P}(L)=L \cdot\left\{\left[\prod_{i=0}^{L-1} \frac{N R E C-i}{N P-i}\right]+\left[\prod_{i=0}^{L-1} \frac{N R E C-i}{N P-i} \cdot \frac{N P-N R E C}{N P-L} \cdot 2 \cdot(n-L-1)\right]+\right. \\
& \left.+\left[\prod_{i=0}^{L-1} \frac{N R E C-i}{N P-i} \cdot \prod_{i=0}^{1} \frac{N P-N R E C-i}{N P-L-i}\right] \cdot \frac{(n-L-1) \cdot(n-L-2)}{2}\right\} \cdot \frac{1}{N R E C},
\end{aligned}
$$

with: $\mathrm{P}(L)=$ the probability that a recurrent point of the TRM belongs to a line of length $L(L=1, \ldots ., n-1)$.

To sum up, if the data series (and, thus, the embedding) were completely random, all the points of the TRM would have the same probability of being recurrent points. Such points would form lines of different sizes according to the probability distribution given in [2]. Considering that $N P=\frac{n \cdot(n-1)}{2}$, this probability distribution only depends on the number of recurrent points, “NREC”, and the number of elements of the embedding, " $n$ ".

In these conditions, the expected value of $L$ will be: $\mathrm{E}[L]=\sum_{L=1}^{n-1} L \cdot P(L)$, and its variance: $\operatorname{Var}[L]=\sum_{L=1}^{n-1}[L-E(L)]^{2} \cdot P(L)$.

This probability distribution is going to be the key tool which will enable us to test whether, in a TRM obtained for a real series, the distribution of points forming line segments differs or not significantly from that which would be expected if the series under study were absolutely random. Concretely, we will propose three test methods, with the null hypothesis in all three cases being that the series under study has been generated from a purely random process. Therefore, rejecting this hypothesis would be evidence that the series presents some deterministic component.

Concretely we propose the following test methods:

1.- The test based on the average value of the random variable $L$ ("Average number of points per line”).

2.- That based on the \%DET.

3.- That based on the average line length, ALL. 


\section{1.- Test based on $\bar{L}$ :}

We define $\bar{L}$ (which we can call "average number of points per line”), in the following terms:

$$
\bar{L}=\frac{1}{N R E C} \cdot \sum_{j} L\left(y_{j}\right)=\sum_{L=1}^{n-1} L \cdot \frac{N R E C_{L}}{N R E C}=\sum_{L=1}^{n-1} L \cdot f(L),
$$

where: $\quad N R E C_{L}=$ Number of recurrent points in lines of length $L$.

$$
f(L)=\text { Proportion of recurrent points in lines of length } L \text {. }
$$

We observe that this statistic is the sample mean of $L$, so it is a good estimator for $E[L]$. Therefore, if the value of this indicator for the series under study is significantly greater than $E[L]$, following the reasoning outlined above, we would conclude that the number of recurrent points on lines parallel to the diagonal would be high enough for us to accept that the series presents some deterministic component. Thus, the null hypothesis would be rejected.

If the data generating process were purely random, we would have NREC points arbitrarily distributed through the TRM, each of which would be associated with a different realization of the random variable $\mathrm{L}$.

In these conditions, it can be easily demonstrated that:

$\mathrm{E}[\bar{L}]=\mathrm{E}[\mathrm{L}]$, and $\operatorname{Var}[\bar{L}]=\frac{\operatorname{Var}[L]}{N R E C}$, so that for a sufficiently high number of recurrent points:

$$
\bar{L} \stackrel{D}{\longrightarrow} N\left(\mathrm{E}[L], \frac{\operatorname{Var}[L]}{N R E C}\right] \text { or equally: } \frac{\bar{L}-E[L]}{\sqrt{\operatorname{Var}[L] / N R E C}} \stackrel{D}{\longrightarrow} N(0,1)
$$

\section{2.- Test based on the \%DET:}

For a purely random data generating process, we can easily obtain the expected $\% D E T$, which we will call $\mathrm{E}[\% D E T]$, from the previous probability distribution. Concretely:

$$
\mathrm{E}[\% D E T]=\sum_{L>1} P(L)=1-P(1)
$$


That is to say that the expected value of $\% D E T$ is equal to the expected proportion of points of the TRM that form lines of a size larger than one or, in other words, equal to one minus the proportion of loose points.

In this case, it would be necessary to test whether the \%DET corresponding to the real series under study differs significantly from E[\%DET]. To do this, we are going to use the Chi-square Test, proposed with the aim of testing whether the distribution from which a data sample is drawn is the same as the theoretical specified distribution.

The chi-square test is defined for the null hypothesis $\mathrm{H}_{0}$ : "the data follow a specified distribution", against the alternative hypothesis Ha: "the data do not follow the specified distribution”.

To apply this test, the following statistic must be constructed:

$$
\chi^{2}=\sum_{i=1}^{L_{m}} \frac{\left(O F_{i}-E F_{i}\right)^{2}}{E F_{i}},
$$

where:

$O F_{i}=$ observed frequency for bin (or category) $i$.

$E F_{i}=$ expected (or theoretical) frequency for bin $i$.

$L_{m}=$ number of bins.

For its application, this test requires a sufficiently high sample size and that all the expected frequencies be at least equal to five. Under these conditions, this statistic follows, approximately, a chi-square distribution with $\left(L_{m}-1\right)$ degrees of freedom.

In our case we only have two bins: ${ }^{11}$ the first of them formed by all the loose points and the second formed by the points forming lines parallel to the main diagonal. The statistic would then be:

$$
\chi^{2}=\frac{(N P D-N R E C \cdot \mathrm{E}[D E T])^{2}}{N R E C \cdot \mathrm{E}[D E T]}+\frac{\{(N R E C-N P D)-N R E C \cdot(1-\mathrm{E}[D E T])\}^{2}}{N R E C \cdot(1-\mathrm{E}[D E T])}
$$

which, if the data generating process were purely random, would follow a chi-square distribution with one degree of freedom. It would then be necessary, therefore, to test whether the sample data respect the theoretical probability distribution (null

\footnotetext{
${ }^{11}$ In this case $\left(L_{m}=2\right)$, the test application requirements are more demanding: concretely, both theoretical frequencies must be at least equal to ten, which in our case will be always fulfilled.
} 
hypothesis), so that rejecting this hypothesis would allow us to conclude that the series presents some deterministic component. We should point out that we will only reject the null hypothesis if the value of \%DET is significantly higher than that corresponding to a purely random process. On the contrary, if \%DET is lower than E[\%DET], although the value of the statistic were high (superior to the pre-established critical point), it would make no sense to reject the null hypothesis.

\section{3.- Test based on $A L L$}

The third statistical procedure that we are going to consider is based on the $A L L$ (“Average Line Length”) which can be defined as the ratio between the total number of recurrent points and the total number of lines ${ }^{12}$

$$
A L L=\frac{N R E C}{N L}=\frac{N R E C}{\sum_{j} \frac{1}{L\left(y_{j}\right)}}=\frac{N R E C}{N R E C \sum_{L=1}^{n-1} \frac{f(L)}{L}}=\frac{1}{\sum_{L=1}^{n-1} \frac{f(L)}{L}},
$$

where: $N L=$ total number of lines in the $T R M$ (of any size, including lines of length 1)

In view of the previous expression, for simplicity's sake, instead of using the $A L L$ we are going to work with its inverse, which we will call $A L L^{-1}$. So:

$$
A L L^{-1}=\frac{1}{A L L}=\frac{1}{N R E C} \cdot \sum_{j} \frac{1}{L\left(y_{j}\right)}=\sum_{L=1}^{n-1} \frac{f(L)}{L}
$$

If the series being studied is purely random, reasoning in terms similar to those we used for the case of the statistic $\bar{L}$, we can conclude that: $\mathrm{E}\left[A L L^{-1}\right]=\mathrm{E}\left[L^{-1}\right]$, $\operatorname{Var}\left[A L L^{-1}\right]=\frac{\operatorname{Var}\left[L^{-1}\right]}{N R E C}$, so that for a sufficiently large number of recurrent points:

$$
\begin{array}{ll}
A L L^{-1} & \stackrel{D}{\longrightarrow} N\left(\mathrm{E}\left[L^{-1}\right], \quad \frac{\operatorname{Var}\left[L^{-1}\right]}{N R E C}\right), \quad \text { or, } \quad \text { equally: } \\
\frac{A L L^{-1}-E\left[L^{-1}\right]}{\sqrt{\operatorname{Var}\left[L^{-1}\right] / N R E C}} \stackrel{D}{\longrightarrow} N(0,1) .
\end{array}
$$

\footnotetext{
${ }^{12}$ The statistics $\bar{L}$ and $A L L$ both increase as the number and the size of the lines parallel to the main diagonal increase. However, while in the latter the average is calculated taking the number of lines as the base, in the former the number of points per line is considered. That is to say that while, to calculate the $A L L$, all the lines are weighted equally, in the case of $\bar{L}$ each line is weighted according to its size (that is, the number of points by which it is formed).
} 
The test procedure would be similar to those of previous paragraphs: to obtain the value of this statistic for the series under study and compare it with the value that would be expected if the recurrent points were randomly distributed through the TRM. Concretely, if $A L L^{-1}$ is significantly lower than $\mathrm{E}\left[A L L^{-1}\right]$ (the value which would be expected if the series being studied were purely random) we would have to reject the null hypothesis, which would be evidence that the series under study presents some deterministic component.

\section{4.-Performance of The Proposed Methods}

To study the performance of the methods proposed above, we are going to apply them to a number of simulated and real series. In the first case, the series under study will be generated from deterministic systems (chaotic or not) or well-known random ones. In the second case we will use the series of US Stock Market returns that, in a previous work (Aparicio et al., 2002) showed evidence of chaotic behaviour.

In the first case, we apply the testing methods to 21 simulated series:

-A non-chaotic deterministic series: that obtained from the sine function.

-Four chaotic deterministic series: those from two discrete systems, Henon and Ikeda, and from two continuous systems, Lorenz and Rossler. For each of the continuous systems two series have been generated using different sampling intervals.

-Eleven random series, using different values of the parameters in some cases.

Tables 2 to 4, corresponding to different sizes of the series, show the values of the three statistics and the p-value or marginal significance level associated with each of them. S1 is the statistic based on the \%DET, S2 is the one based on the average number of points per line, and S3 is based on the average line length. In all cases a radius " $\mathrm{r}$ " has been fixed to assure a percentage of recurring points (\%REC) of around 10\%:

\section{(Tables 2 to 4 about here)}

Those cells of the statistic based on \%DET in which there are no p-values are cases in which the \%DET is lower than $\mathrm{E}[\% \mathrm{DET}]$. In these cases, as we established previously, the null hypothesis cannot be rejected.

From these results it can be observed that: 
-In the case of the deterministic systems, the three methods would lead to rejecting the null hypothesis for whatever level of significance.

-For the random systems, the three methods also lead to the correct result, that is, not to rejecting the null hypothesis. The only exception is that corresponding to the series generated with Student's t system with four degrees of freedom, for $n=500$, and with a level of significance of 5\%.

In short, the methods proposed seem to work well. However, to obtain further evidence against the null hypothesis, we will only reject it when the three statistics proposed give this result.

Nevertheless, as we are using only one outcome of each series, we understand that, to obtain more robust results, it would be necessary to replicate the experiment a high number of times.

In Table 5 are shown the results corresponding to 1000 replications of some of the systems for different sample sizes. We are trying to measure the level of success of the proposed tests: that is to say, the percentage of times the null hypothesis would not be rejected when the series is generated by a random system or the percentage of times it would be rejected when the series is generated by a deterministic system ${ }^{13}$. In all cases the two most habitual levels of significance are considered (5\% and 1\%).

\section{(Table 5 about here)}

The results of Table 5 are highly satisfactory. The percentage of success is very high in all cases. We can observe that when the system that generates the series is random, the percentage of failures is very close to the nominal level of the tests (0.05 and 0.01), while if the system that generates the series is deterministic, the percentage of success (or power of the tests) is $100 \%$.

For the deterministic systems we have considered two different values of the embedding: $m=3$ (equal or superior to the real dimension of the systems considered) and $\mathrm{m}=1$, with the same results in both cases. The values in brackets are those corresponding to $\mathrm{m}=1$

Furthermore, we can observe that even with small sample sizes the behaviour of the tests satisfactory.

\footnotetext{
${ }^{13}$ The simulations of the Henon and Lorenz deterministic systems have been carried out considering different values of the parameters of both systems (making sure, nevertheless, that in all the cases their chaotic nature is maintained).
} 
Given the good results obtained when the series is generated in a deterministic system, it may be interesting to analyse the influence of noise on the results of the tests. In other words, if the series were affected by different levels of noise, above which levels of noise would the distortion be significant enough to modify their originally deterministic character. Specifically, we obtain results for the series with different amounts of gaussian noise added, with zero mean, and standard deviation expressed as percentage of the corresponding noise-free data.

\section{(Table 6 about here)}

The results presented in Table 6 allow us to draw the following conclusions. In the first place, as was to be expected, the percentage of success is lower as the proportion of noise added increases. ${ }^{14}$ Likewise, the consideration of an embedding lower than the original dimension of the system worsens the results in all cases. This is an immediate consequence of the existence of "false neighbours" that in a dimension inferior to that of the system would act as noise. Secondly, the percentage of success is elevated even for very high levels of noise, which means that the proposed method adequately reflects the underlying determinism in the series. Consequently, if, when it is applied to a real series, we cannot reject the null hypothesis of randomness, we should be able to trust in the purely stochastic nature of the series.

As we said at the beginning of this section, we consider it interesting to apply this procedure to real series. To this end, we are going to use the series employed in a previous work (Aparicio et al., 2002), namely, five series of US Stock Market daily returns from January 2, 1990 to September 30, 1998 (with a total of 2211 observations each): S\&P 500 index, S\&P 100 index, NYSE industrial index, and the series of share prices of the companies General Motors Corp. (GM) and Chase Manhattan Corp. (CMB).

In that work, applying the "Nearest Neighbour Method", we found evidence of underlying chaotic behaviour in the five series. Furthermore, for a time delay equal to one, we estimated the embedding dimensions of each of them.

Table 7 shows the results obtained from the application of the three tests to the series mentioned.

\footnotetext{
${ }^{14}$ Based on this fact Matassini et al. (2002) design an algorithm to identify and filter the noise level present in the time series.
} 


\section{(Table 7 about here)}

For each series we have obtained results for an embedding equal to one and for the embedding estimated in the work mentioned. Observing the value of the statistics, we can highlight that in all cases the null hypothesis of randomness is rejected, which confirms the results obtained with the "Nearest Neighbour Method". From the values of the statistics it could be thought that the selection of the embedding is not important, because the deterministic character of the series is being captured for all of them. However, a deeper analysis of the last three columns of Table 7 shows that this is not completely true: the values of the three indicators (\%DET, $\bar{L}$ and $A L L$ ) are clearly lower in the cases in which the embedding dimension is equal to one, not being very different to those that would be expected if the series were purely random $(\% D E T \approx$ $19.1 \%, \bar{L} \approx 1.23$ and $A L L \approx 1.11$ ). This behaviour, just as occurred in the previous simulation experiment, shows how the influence of false neighbours distorts the results when the embedding dimension is lower than the estimated one.

\section{5.-Summary and Conclusions}

In this paper, we proposed three test procedures for detecting underlying determinism in series that, because of their behaviour, could be considered as apparently random. We used an interesting graphic tool, the Recurrence Plot, designed by Eckman in 1987 and whose obtaining and interpretation is simple and intuitive. In particular, our objective is to go deeper into the approach called RQA, "Recurrence quantification analysis” initiated a decade ago by Zbilut and Webber to formalise in analytical, and thus, more rigorous terms, the results shown by RPs. Within this line of work, and starting from the geometric structure of the RP and, in particular, the number of points that form lines parallel to the main diagonal, we designed three test procedures. One of them is based on the number of points on the lines (\%DET), another on the average number of points per line, and a third on the average line length.

To evaluate these tests, we applied them to a group of simulated series, both random and deterministic, with the following conclusions. Firstly, we must highlight the general good behaviour of the tests, with percentages of success always higher than $90 \%$ and even of $100 \%$ on many occasions. When success means the non rejection of the null hypothesis, the proportion of failures is very near the nominal level of the tests, 
while if success means the rejection of the null hypothesis, the percentage of success or power of the test is $100 \%$. Secondly, this good behaviour is maintained even for small sample sizes (100 observations). Lastly, for deterministic series affected by noise, the deterministic component is detected even for high levels of noise.

Finally, we apply these procedures to five series of US Stock Market daily returns with which, in a previous work, we obtained evidence of chaotic behaviour. The high deterministic component that we found in them ratifies the result obtained in the above-mentioned work and is an argument in favour of the use of the procedures proposed.

To advance in the development of these tests to detect chaotic behaviour (not only deterministic) and to establish the dimension of the systems constitute future lines of investigation.

\section{References}

Aparicio, T., Pozo, E. And Saura, D. (2002) The Nearest Neighbour Method As A Test For Detecting Complex Dynamics In Financial Series. An Empirical Application. Applied Financial Economics 12, 517-525.

Atay, F.M., And Altintas, Y. (1999) Recovering Smooth Dynamics From Time Series With The Aid Of Recurrence Plots. Physical Review E 59, 6593-6598.

Bradley, E. (2002) Time-Series Analysis. In Intelligent Data Analysis: An Introduction (Eds M. Berthold And D. Hand). Berlin: Springer Verlag, Ch. 5.

Buzug, Th., Reimers, T. And Pfister, G. (1990) Optimal Reconstruction Of Strange Attractors From Purely Geometrical Arguments. Europhysics Letters 13, 605-610.

Casdagli, M.C. (1997) Recurrence Plots Revisited. Physica D 108, 12-44.

Eckmann, J.P., Kamphorst, S. And Ruelle, D. (1987) Recurrence Plots Of Dynamical Systems. Europhysics Letters 4, 973-977.

Gao, J. And Cai, H. (2000) On The Structures And Quantification Of Recurrence Plots. Physics Letters A 270, 75-87.

Haucke, H., Ecke, R.E. And Wheatley, J.C. (1986) Dimension And Entropy For Quasiperiodic And Chaotic Convection. In Dimensions And Entropies In Chaotic Systems (Ed G. Mayer-Kress). Berlin: Springer-Verlag, 198-206. 
Iwanski, J. And Bradley, E. (1998) Recurrence Plot Analysis: To Embed Or Not To Embed?. Chaos 8, 861-871.

Marwan N., Wessel, N., Meyerfeldt, U., Schirdewan, A. And Kurths, J. (2002) Recurrence Plot Based Measures Of Complexity And Its Application To Heart Rate Variability Data. Physical Review E 66, 026702.

Matassini, L., Kantz, H., Holyst, J. And Hegger, R. (2002) Optimizing Of Recurrence Plots For Noise Reduction. Physical Review E 65, 021102.

Mayfield, E.S., And Mizrach, B. (1992) On Determining The Dimension Of Real-Time StockPrice Data. Journal Of Business Economics And Statistics 10, 367-74.

Packard, N., Crutchfield, J.P., Farmer, J.D. And Shaw, R.S. (1980) Geometry From A Time Series. Physical Review Letters 45, 712-716.

Takens, F. (1981) Detecting Strange Attractors In Turbulence. In Dynamical Systems And Turbulence (Eds D. Rand And L. Young). Berlin: Springer-Verlag.

Thiel, M., Romano, M.C., Read, P.L. And Kurths, J. (2003) Analytical Description Of Recurrence Plots Of White Noise And Chaotic Proceses. Jzv. Vuzov - Applied Nonlinear Dynamics, 11.

Thiel, M., Romano, M.C., Read, P.L. And Kurths, J. (2004) Estimation Of Dynamical Invariants Without Embedding By Recurrence Plots. Chaos 14, 234-243.

Webber Jr., C.L., And Zbilut, J.P. (1994) Dynamical Assessment Of Physiological Systems And States Using Recurrence Plot Strategies. Journal Of Applied Physiology 76, 965-973.

Zbilut, J.P., Giuliani, A., And Webber Jr., C.L. (2000) Recurrence Quantification Analysis As An Empirical Test To Distinguish Relatively Short Deterministic Versus Random Number Series. Physics Letters A 267, 174-178.

Zbilut, J.P. And Webber Jr., C.L. (1992) Embeddings And Delays As Derived From Quantification Of Recurrence Plots. Physics Letters A 171, 199-203. 


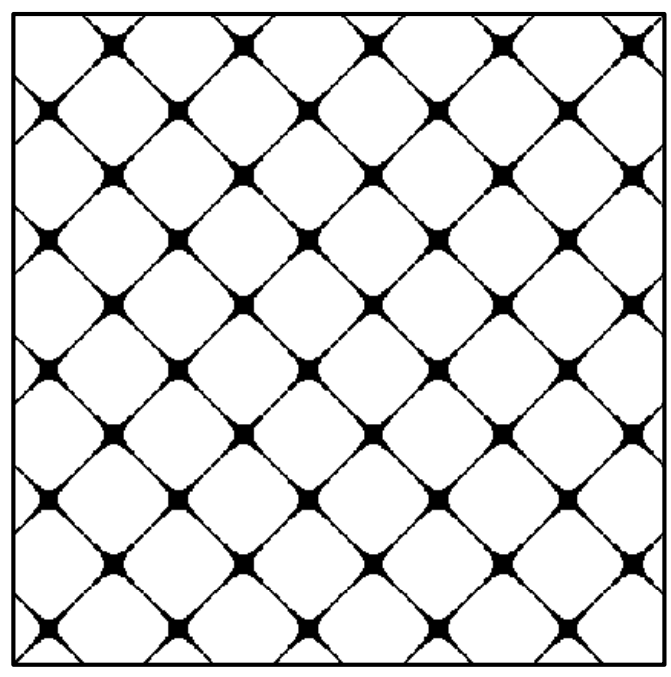

(a)

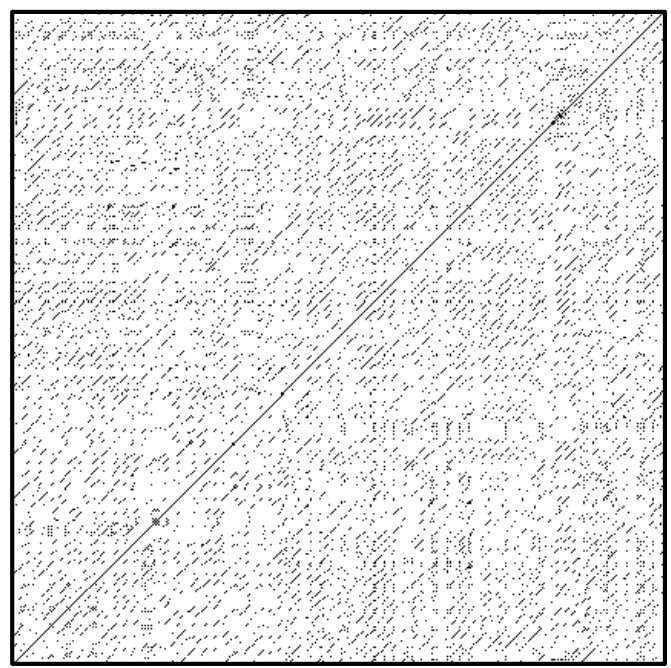

(c)

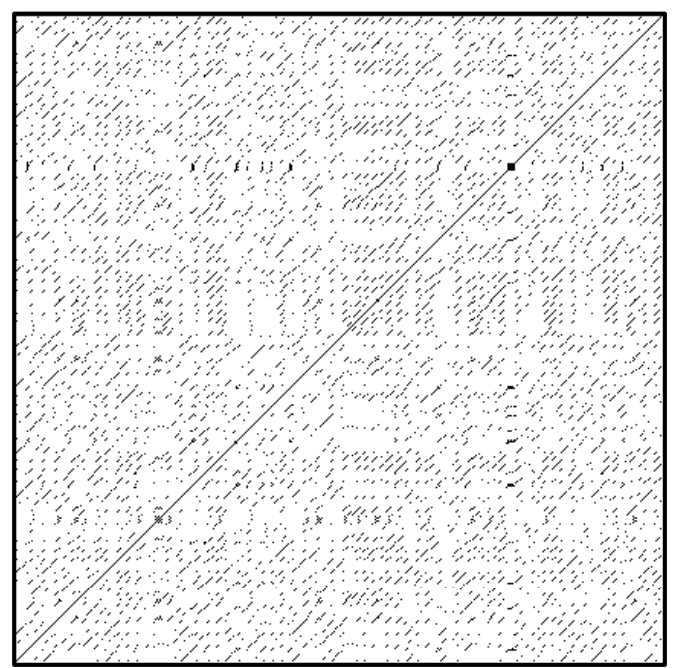

(b)

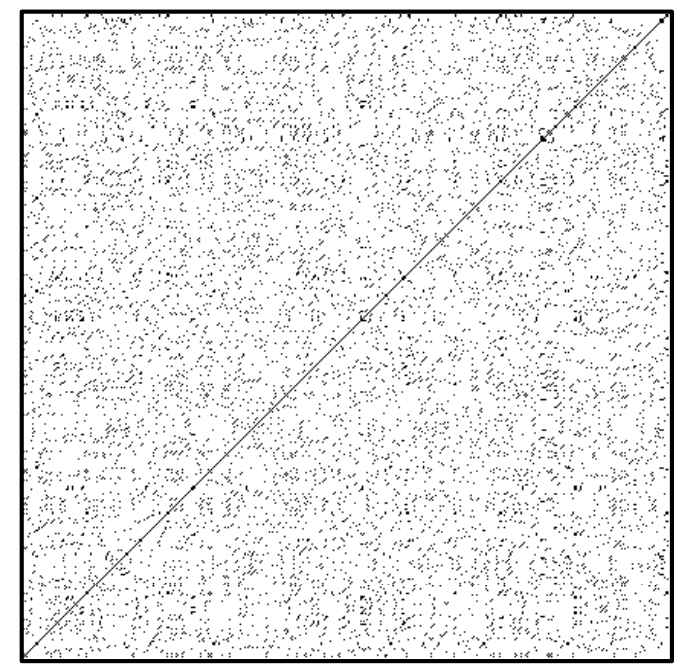

(d)

Figure 1: Recurrence plots constructed from 500 data generated by:

(a)-The sine function $(\mathrm{m}=1, \mathrm{t}=1)$.

(b)-The Henon attractor $(\mathrm{m}=3, \mathrm{t}=1)$.

(c)-The Henon attractor with a $25 \%$ uniform noise added $(m=3, t=1)$.

(d)-A white noise $(\mathrm{m}=1, \mathrm{t}=1)$.

( $\mathrm{m}$ = embedding dimension; $\mathrm{t}=$ time delay) 


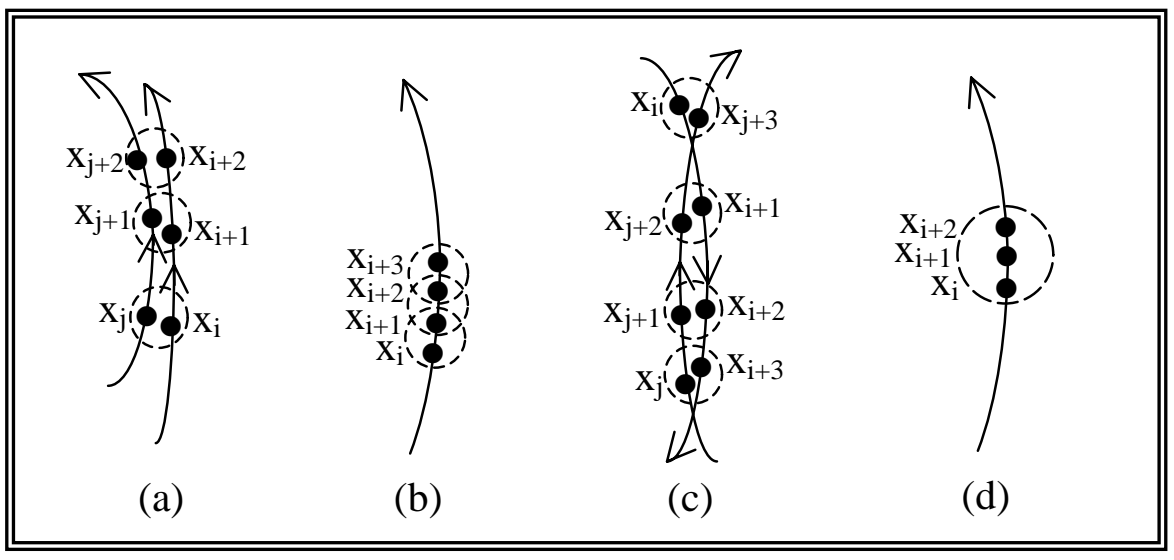

Figure 2: Patterns of the RP related to the three kinds of lines:
(a) and (b): Lines parallel to the main $45^{\circ}$ diagonal.
(c): Lines parallel to the $135^{\circ}$ diagonal.
(d): Horizontal and vertical lines. 
Table 1: Values of \%DET and $A L L$ corresponding to the RPs of Figure 1

\begin{tabular}{|lcc|}
\cline { 2 - 3 } \multicolumn{1}{c|}{} & \%DET & ALL \\
\hline \hline Sine & 95.91 & 5.37 \\
\hline Henon & 88.21 & 2.98 \\
\hline Henon with Noise & 81.13 & 2.34 \\
\hline White Noise & 20.14 & 1.12 \\
\hline
\end{tabular}




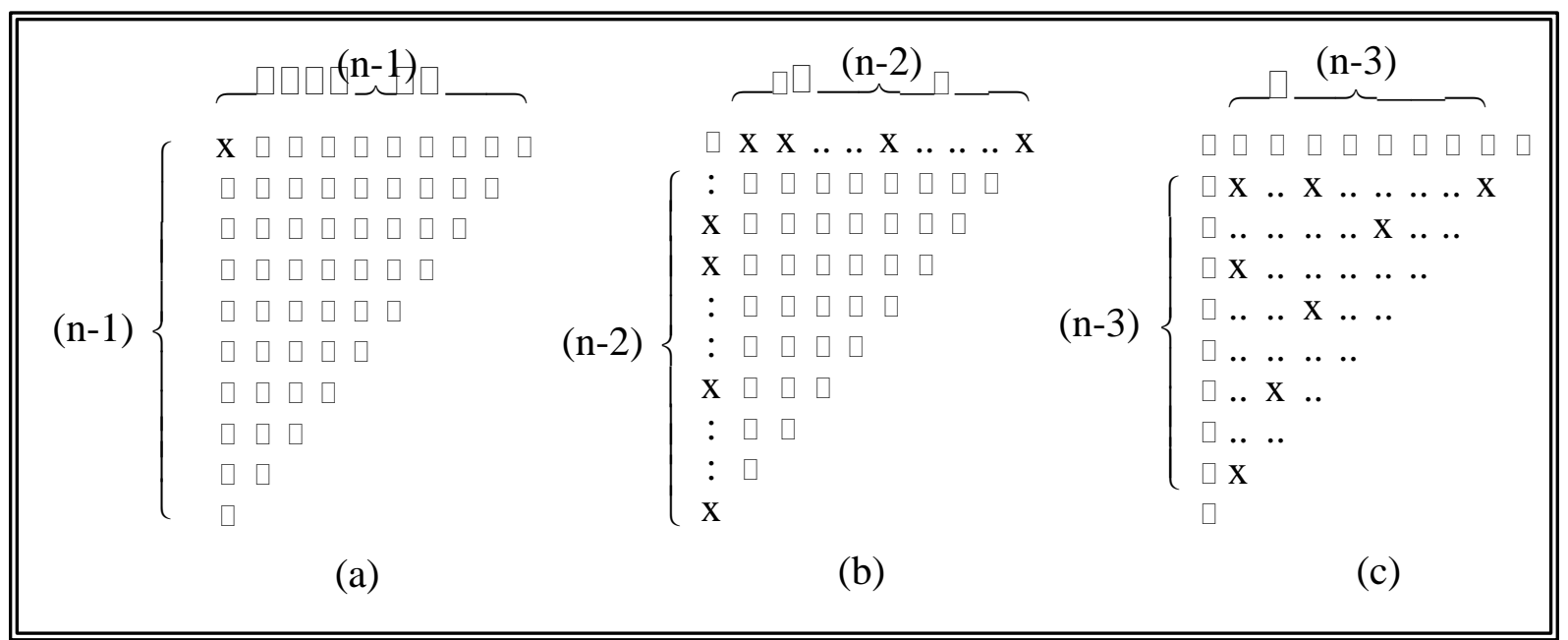

Figure 3: Lines parallel to the main diagonal: Average line length (Length 1 lines) 1. (a): If the element $(1,1)$ of the TRM is a recurrent point it would form a line of length

(b): Elements of the first row and the first column of the TRM, except $(1,1)$.

(c): Interior elements of the TRM. 


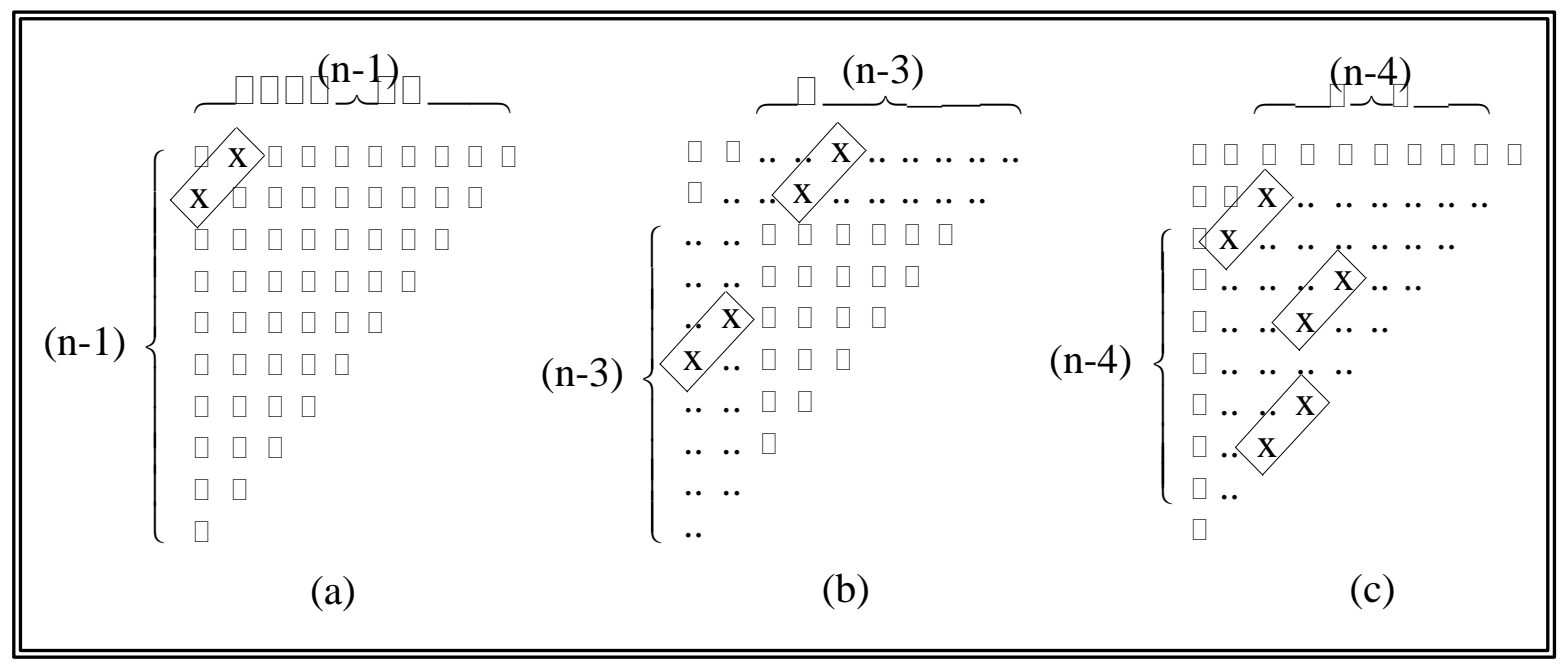

Figure 4: Lines parallel to the main diagonal: Average line length (Length 2 lines) (a): Lines of length 2 formed by the elements $(1,2)$ and $(2,1)$ of the TRM.

(b): Lines of length 2 containing elements of the first row and the first column of the TRM.

(c): Lines of length 2 formed by interior elements of the TRM. 
Table 2: Values of the statistics and corresponding p-values for the series $(\mathrm{n}=100)$.

\begin{tabular}{|lcccccc|}
\hline Series & S1 & p-value & S2 & p-value & S3 & p-value \\
\hline \hline Sine & 1917.26 & 0.00 & 1225.41 & 0.00 & -75.12 & 0.00 \\
\hline Henon & 1437.81 & 0.00 & 139.32 & 0.00 & -56.12 & 0.00 \\
\hline Ikeda & 917.53 & 0.00 & 140.61 & 0.00 & -49.06 & 0.00 \\
\hline Lorenz1 & 1877.64 & 0.00 & 434.70 & 0.00 & -75.58 & 0.00 \\
\hline Lorenz2 & 998.78 & 0.00 & 178.94 & 0.00 & -49.99 & 0.00 \\
\hline Rossler1 & 2055.27 & 0.00 & 1496.39 & 0.00 & -83.61 & 0.00 \\
\hline Rossler2 & 1584.48 & 0.00 & 643.08 & 0.00 & -62.33 & 0.00 \\
\hline Beta(2,1) & 0.51 & - & -1.26 & 0.90 & 0.92 & 0.82 \\
\hline Bin(1000,0.5) & 11.07 & - & -4.18 & 1 & 3.71 & 1 \\
\hline Bin(1000,0.1) & 1.53 & - & -0.65 & 0.74 & 1.08 & 0.86 \\
\hline Normal(0,1) & 1.53 & - & -1.76 & 0.96 & 1.44 & 0.92 \\
\hline Normal(5,2) & 0.17 & 0.68 & -1.11 & 0.87 & 0.65 & 0.74 \\
\hline Expon(5) & 0.09 & - & -0.87 & 0.81 & 0.50 & 0.69 \\
\hline Gamma(1,2) & 0.55 & 0.46 & 1.13 & 0.13 & -0.95 & 0.17 \\
\hline Geom(0.3) & 1 & - & -1.71 & 0.96 & 2.15 & 0.98 \\
\hline Hprg(120,50,50) & 0.98 & 0.32 & -0.04 & 0.52 & 0.72 & 0.77 \\
\hline Poisson(10) & 0.03 & 0.87 & 0.26 & 0.40 & -0.17 & 0.43 \\
\hline Rayleigh(0.5) & 0.27 & 0.60 & 1.17 & 0.12 & -0.80 & 0.21 \\
\hline t(2) & 2.66 & 0.10 & -1.57 & 0.94 & 1.69 & 0.95 \\
\hline t(4) & 2.06 & 0.15 & -0.10 & 0.54 & 0.97 & 0.83 \\
\hline Uniform & 1.74 & - & -1.02 & 0.85 & 1.20 & 0.88 \\
\hline
\end{tabular}


Table 3: Values of the statistics and corresponding p-values for the series $(n=500)$.

\begin{tabular}{|lcccccc|}
\hline Series & S1 & p-value & S2 & p-value & S3 & p-value \\
\hline \hline Sine & 47491.37 & 0.00 & 22407.69 & 0.00 & -376.63 & 0.00 \\
\hline Henon & 36331.52 & 0.00 & 936.96 & 0.00 & -296.26 & 0.00 \\
\hline Ikeda & 33374.40 & 0.00 & 1172.45 & 0.00 & -299.35 & 0.00 \\
\hline Lorenz1 & 46479.17 & 0.00 & 2853.55 & 0.00 & -364.68 & 0.00 \\
\hline Lorenz2 & 26148.14 & 0.00 & 730.95 & 0.00 & -247.73 & 0.00 \\
\hline Rossler1 & 52913.69 & 0.00 & 26696.39 & 0.00 & -448.71 & 0.00 \\
\hline Rossler2 & 43582.58 & 0.00 & 4772.95 & 0.00 & -345.90 & 0.00 \\
\hline Beta(5,0.2) & 1.89 & 0.17 & -0.29 & 0.62 & 0.97 & 0.83 \\
\hline Bin(1000,0.5) & 14.12 & - & -3.94 & 1.00 & 3.88 & 1.00 \\
\hline Bin(1000,0.1) & 3.96 & - & -2.17 & 0.99 & 2.11 & 0.98 \\
\hline Normal(0,1) & 1.07 & 0.30 & 0.13 & 0.45 & 0.64 & 0.74 \\
\hline Normal(5,2) & 0.11 & 0.74 & -0.26 & 0.60 & -0.23 & 0.41 \\
\hline Expon(5) & 0.14 & 0.71 & 0.52 & 0.30 & -0.44 & 0.33 \\
\hline Gamma(1,2) & 23.66 & - & -4.69 & 1.00 & 4.85 & 1.00 \\
\hline Geom(0.3) & 8.17 & - & -2.59 & 1.00 & 2.82 & 1.00 \\
\hline Hprg(120,50,50) & 2.25 & 0.13 & 0.15 & 0.44 & -1.09 & 0.14 \\
\hline Poisson(10) & 0.04 & 0.84 & 0.18 & 0.43 & -0.22 & 0.41 \\
\hline Rayleigh(0.5) & 0.72 & 0.40 & 0.61 & 0.27 & -0.80 & 0.21 \\
\hline $\mathrm{t}(2)$ & 0.31 & 0.57 & 1 & 0.16 & -0.87 & 0.19 \\
\hline $\mathrm{t}(4)$ & 5.22 & $\mathbf{0 . 0 2}$ & 1.61 & 0.06 & -2.06 & $\mathbf{0 . 0 2}$ \\
\hline Uniform & 0.01 & 0.91 & -0.73 & 0.77 & 0.23 & 0.59 \\
\hline & & & & & & \\
\hline
\end{tabular}


Table 4: Values of the statistics and corresponding p-values for the series $(\mathrm{n}=1000)$.

\begin{tabular}{|lcccccc|}
\hline Series & S1 & p-value & S2 & p-value & S3 & p-value \\
\hline Sine & 189824.66 & 0.00 & 89474.44 & 0.00 & -755.28 & 0.00 \\
\hline Henon & 147756.70 & 0.00 & 1970.29 & 0.00 & -599.13 & 0.00 \\
\hline Ikeda & 124370.39 & 0.00 & 2246.35 & 0.00 & -580.05 & 0.00 \\
\hline Lorenz1 & 181595.01 & 0.00 & 7296.72 & 0.00 & -719.88 & 0.00 \\
\hline Lorenz2 & 106077.70 & 0.00 & 1520.97 & 0.00 & -500.02 & 0.00 \\
\hline Rossler1 & 212160.60 & 0.00 & 47839.18 & 0.00 & -890.06 & 0.00 \\
\hline Rossler2 & 174576.37 & 0.00 & 11583.48 & 0.00 & -690.82 & 0.00 \\
\hline Beta(5,0.2) & 0.38 & 0.54 & 1.30 & 0.10 & -0.77 & 0.22 \\
\hline Bin(1000,0.5) & 0.10 & 0.75 & -0.65 & 0.74 & 0.39 & 0.65 \\
\hline Bin(1000,0.1) & 1.38 & - & -1.70 & 0.96 & 1.42 & 0.92 \\
\hline Normal(0,1) & 5.04 & - & -1.99 & 0.98 & 2.23 & 0.99 \\
\hline Normal(5,2) & 6.39 & - & -2.20 & 0.99 & 2.53 & 0.99 \\
\hline Expon(5) & 0.55 & 0.46 & 1.13 & 0.13 & -0.95 & 0.17 \\
\hline Gamma(1,2) & 8.93 & - & -5.13 & 1.00 & 3.88 & 1.00 \\
\hline Geom(0.3) & 0.14 & 0.71 & 1.15 & 0.13 & -0.05 & 0.48 \\
\hline Hprg(120,50,50) & 5.73 & - & -2.88 & 1.00 & 2.59 & 0.99 \\
\hline Poisson(10) & 0.55 & 0.46 & -0.18 & 0.57 & -0.46 & 0.32 \\
\hline Rayleigh(0.5) & 1.68 & 0.20 & -2.15 & 0.98 & 1.59 & 0.94 \\
\hline $\mathrm{t}(2)$ & 15.10 & - & -3.78 & 1.00 & 3.98 & 1.00 \\
\hline $\mathrm{t}(4)$ & 0.41 & 0.52 & 0.18 & 0.43 & -0.51 & 0.30 \\
\hline Uniform & 0.07 & - & -1.14 & 0.87 & 0.55 & 0.71 \\
\hline
\end{tabular}


Table 5: Percentage of success of the proposed tests in deterministic and random series (number of replications $=1000$ ).

\begin{tabular}{|lccc|}
\hline Series & $\mathbf{n}$ & \%Success (0.05) & \%Success (0.01) \\
\hline \hline Normal(0,1) & 100 & 93.1 & 95.8 \\
\hline Normal(0,1) & 500 & 92.9 & 96.1 \\
\hline Normal(0,1) & 1000 & 93.8 & 97.1 \\
\hline Uniform & 100 & 92.4 & 97.3 \\
\hline Uniform & 500 & 93.4 & 97.4 \\
\hline Uniform & 1000 & 94.5 & 97.4 \\
\hline Henon & 100 & $100(100)$ & $100(100)$ \\
\hline Henon & 500 & $100(100)$ & $100(100)$ \\
\hline Henon & 1000 & $100(100)$ & $100(100)$ \\
\hline Lorentz & 100 & $100(100)$ & $100(100)$ \\
\hline Lorentz & 500 & $100(100)$ & $100(100)$ \\
\hline Lorentz & 1000 & $100(100)$ & $100(100)$ \\
\hline
\end{tabular}


Table 6: Percentage of success of the proposed tests in deterministic series with noise added

(number of replications $=1000$ ).

\begin{tabular}{|cccc|}
\hline Series & n & \%Success (0.05) & \%Success (0.01) \\
\hline \hline Henon+25\%noise & 100 & $100(100)$ & $100(99.9)$ \\
\hline Henon+50\%noise & 100 & $100(74.1)$ & $100(60.9)$ \\
\hline Henon+75\%noise & 100 & $99.7(34.5)$ & $99.3(21.6)$ \\
\hline Henon+100\%noise & 100 & $84(16.8)$ & $74(7.8)$ \\
\hline Henon+125\%noise & 100 & $49.8(12.5)$ & $37.3(5.9)$ \\
\hline Henon+150\%noise & 100 & $30.8(8.5)$ & $19.4(3.3)$ \\
\hline Henon+175\%noise & 100 & $20(7.8)$ & $11.9(2.8)$ \\
\hline Henon+200\%noise & 100 & $10.2(6.6)$ & $7.3(3.2)$ \\
\hline Lorentz +25\%noise & 100 & $100(100)$ & $100(100)$ \\
\hline Lorentz +50\%noise & 100 & $100(98.4)$ & $100(96.9)$ \\
\hline Lorentz +75\%noise & 100 & $97.8(66.9)$ & $93.9(53.3)$ \\
\hline Lorentz +100\%noise & 100 & $82.2(34.5)$ & $71.2(21.2)$ \\
\hline Lorentz +125\%noise & 100 & $66.2(18.3)$ & $52.4(8.8)$ \\
\hline Lorentz +150\%noise & 100 & $38.2(13.6)$ & $25.7(7.5)$ \\
\hline Lorentz +175\%noise & 100 & $27.6(12.1)$ & $18.9(6.1)$ \\
\hline Lorentz +200\%noise & 100 & $19.6(8.5)$ & $10.8(4.1)$ \\
\hline
\end{tabular}


Table 7: Results of the application of the tests to five series of US Stock Market daily returns $(\% \mathrm{REC} \approx 10 \%$ in all cases).

\begin{tabular}{|l|c|c|c|c|c|c|c|c|c|c|}
\hline Series & $\mathbf{m}$ & S1 & p-value & S2 & p-value & S3 & p-value & \%DET & $\overline{\boldsymbol{L}}$ & $\boldsymbol{A L L}$ \\
\hline \multirow{2}{*}{ SP500 } & 1 & 210.88 & 0 & 16.92 & 0 & -15.58 & 0 & 20.65 & 1.24 & 1.12 \\
\cline { 2 - 10 } & 6 & $7.5 \mathrm{e}^{5}$ & 0 & $4.9 \mathrm{e}^{3}$ & 0 & $-1.3 \mathrm{e}^{3}$ & 0 & 88.03 & 6.23 & 3.15 \\
\hline \multirow{2}{*}{ SP100 } & 1 & 165.02 & 0 & 15.90 & 0 & -14.02 & 0 & 20.40 & 1.24 & 1.12 \\
\cline { 2 - 11 } & 6 & $7.6 \mathrm{e}^{5}$ & 0 & $4.9 \mathrm{e}^{3}$ & 0 & $-1.3 \mathrm{e}^{3}$ & 0 & 88.25 & 6.16 & 3.16 \\
\hline \multirow{2}{*}{ NYSE } & 1 & 165.02 & 0 & 15.90 & 0 & -14.01 & 0 & 20.40 & 1.24 & 1.12 \\
\cline { 2 - 11 } & 3 & $4.1 \mathrm{e}^{5}$ & 0 & $1.6 \mathrm{e}^{3}$ & 0 & $-8.6 \mathrm{e}^{2}$ & 0 & 69.56 & 2.88 & 1.86 \\
\hline GM & 1 & 51.91 & 0 & 9.88 & 0 & -8.26 & 0 & 19.05 & 1.23 & 1.11 \\
\hline & 2 & $1.8 \mathrm{e}^{5}$ & 0 & 722 & 0 & -519 & 0 & 53.80 & 1.96 & 1.46 \\
\hline CMB & 1 & 831.30 & 0 & 37.73 & 0 & -32.23 & 0 & 22.48 & 1.28 & 1.13 \\
\hline & 4 & $5.9 \mathrm{e}^{5}$ & 0 & $2.6 \mathrm{e}^{3}$ & 0 & $-1.1 \mathrm{e}^{3}$ & 0 & 80.25 & 3.89 & 2.33 \\
\hline
\end{tabular}




\section{Documentos de Trabajo}

\section{Facultad de Ciencias Económicas y Empresariales. Universidad de Zaragoza.}

2002-01: "Evolution of Spanish Urban Structure During the Twentieth Century". Luis Lanaspa, Fernando Pueyo y Fernando Sanz. Department of Economic Analysis, University of Zaragoza.

2002-02: “Una Nueva Perspectiva en la Medición del Capital Humano”. Gregorio Giménez y Blanca Simón. Departamento de Estructura, Historia Económica y Economía Pública, Universidad de Zaragoza.

2002-03: “A Practical Evaluation of Employee Productivity Using a Professional Data Base”. Raquel Ortega. Department of Business, University of Zaragoza.

2002-04: "La Información Financiera de las Entidades No Lucrativas: Una Perspectiva Internacional”. Isabel Brusca y Caridad Martí. Departamento de Contabilidad y Finanzas, Universidad de Zaragoza.

2003-01: “Las Opciones Reales y su Influencia en la Valoración de Empresas”. Manuel Espitia y Gema Pastor. Departamento de Economía y Dirección de Empresas, Universidad de Zaragoza.

2003-02: "The Valuation of Earnings Components by the Capital Markets. An International Comparison”. Susana Callao, Beatriz Cuellar, José Ignacio Jarne and José Antonio Laínez. Department of Accounting and Finance, University of Zaragoza.

2003-03: "Selection of the Informative Base in ARMA-GARCH Models". Laura Muñoz, Pilar Olave and Manuel Salvador. Department of Statistics Methods, University of Zaragoza.

2003-04: "Structural Change and Productive Blocks in the Spanish Economy: An Imput-Output Analysis for 1980-1994”. Julio Sánchez Chóliz and Rosa Duarte. Department of Economic Analysis, University of Zaragoza.

2003-05: "Automatic Monitoring and Intervention in Linear Gaussian State-Space Models: A Bayesian Approach”. Manuel Salvador and Pilar Gargallo. Department of Statistics Methods, University of Zaragoza.

2003-06: "An Application of the Data Envelopment Analysis Methodology in the Performance Assessment of the Zaragoza University Departments”. Emilio Martín. Department of Accounting and Finance, University of Zaragoza.

2003-07: "Harmonisation at the European Union: a difficult but needed task". Ana Yetano Sánchez. Department of Accounting and Finance, University of Zaragoza.

2003-08: "The investment activity of spanish firms with tangible and intangible assets". Manuel Espitia and Gema Pastor. Department of Business, University of Zaragoza. 
2004-01: "Persistencia en la performance de los fondos de inversión españoles de renta variable nacional (1994-2002)”. Luis Ferruz y María S. Vargas. Departamento de Contabilidad y Finanzas, Universidad de Zaragoza.

2004-02: "Calidad institucional y factores político-culturales: un panorama inter.nacional por niveles de renta”. José Aixalá, Gema Fabro y Blanca Simón. Departamento de Estructura, Historia Económica y Economía Pública, Universidad de Zaragoza.

2004-03: "La utilización de las nuevas tecnologías en la contratación pública”. José $\mathrm{M}^{\mathrm{a}}$ Gimeno Feliú. Departamento de Derecho Público, Universidad de Zaragoza.

2004-04: "Valoración económica y financiera de los trasvases previstos en el Plan Hidrológico Nacional español”. Pedro Arrojo Agudo. Departamento de Análisis Económico, Universidad de Zaragoza. Laura Sánchez Gallardo. Fundación Nueva Cultura del Agua.

2004-05: "Impacto de las tecnologías de la información en la productividad de las empresas españolas”. Carmen Galve Gorriz y Ana Gargallo Castel. Departamento de Economía y Dirección de Empresas. Universidad de Zaragoza.

2004-06: "National and International Income Dispersión and Aggregate Expenditures”. Carmen Fillat. Department of Applied Economics and Economic History, University of Zaragoza. Joseph Francois. Tinbergen Institute Rotterdam and Center for Economic Policy Resarch-CEPR.

2004-07: “Targeted Advertising with Vertically Differentiated Products”. Lola Esteban and José M. Hernández. Department of Economic Analysis. University of Zaragoza.

2004-08: "Returns to education and to experience within the EU: are there differences between wage earners and the self-employed?”. Inmaculada García Mainar. Department of Economic Analysis. University of Zaragoza. Víctor M. Montuenga Gómez. Department of Business. University of La Rioja

2005-01: "E-government and the transformation of public administrations in EU countries: Beyond NPM or just a second wave of reforms?”. Lourdes Torres, Vicente Pina and Sonia Royo. Department of Accounting and Finance.University of Zaragoza

2005-02: "Externalidades tecnológicas internacionales y productividad de la manufactura: un análisis sectorial”. Carmen López Pueyo, Jaime Sanau y Sara Barcenilla. Departamento de Economía Aplicada. Universidad de Zaragoza.

2005-03: "Detecting Determinism Using Recurrence Quantification Analysis: Three Test Procedures”. María Teresa Aparicio, Eduardo Fernández Pozo and Dulce Saura. Department of Economic Analysis. University of Zaragoza. 